\title{
太阳和恒星的磁場
}

\author{
叶式煇
}

(中国科学院紫金山天交台)

从二十世纪初期以来, 天体物理学经历 了巨大而深刻的变化。大型望远镜和太阳塔 的制作、射电天文学的诞生、星际航行时代 的来临以及一系列新技术的应用, 都使这门 学科的领域大为扩充。以丰富的观测资料为 依据，恆星大气物理、恆星內部结构、太 阳活动区物理等的完整而严谨的理论应运 而生。我们目前对天体的物态、成分、结 构、起源和演化的认识, 虽然还是很不够 的，但与本世纪之初相比，已经不可同日而 语了。必须谈到, 在天体物理牛个多世纪来 的发展中，有一个重要的方面，它的意义已 经变得日益明显。这就是天体磁场的研究。 我们知道, 仅仅除掉象地球这样的行星外, 宇宙中绝大部分物质 (太阳、恆星、星云以 及星际介质)都是等离子体*。它们的物态、 运动和演化都与电磁场息息相关。因此, 要 深入了解天体的物理性质, 必须研究它们的 磁场。实际上，六十多年来已有许多天文工 作者致力于这方面的探索, 并取得了不少杰 出的成就。尤其是晚近十余年来, 由于观测 技术的精进, 许多错综复杂的现象不断被发 现, 新的理论层出不穷。这个迅速扩充和日 益丰富多采的工作领域, 值得介绍给广大的 读者。

\section{一 历史回厥}

早在四、五千年前, 我们的祖先就发明 了指南針, 为地球磁场的研究揭开了序幕。 但是天体磞场的研究却是近代的事。虽然在 19 世纪已经有人认为恆星具有和地球相似 的磁性, 但正式的测量和研究仅发韧于本世 纪初期。迄今为止, 大部分工作都是对太阳 进行的。这不难理解，因为对栖息在地球上 的人类来说, 太阳是最大、最亮、也是唯一 能看到表面细节的恆星。从 1908 年开始, 海耳 (Hale G. E.) 和尼科耳孙 (Nicholson S. B. ) 在威耳逊山天文台测量太阳黑子的磁 场，得到了肯定的结果，料发现了好些有趣 的规律。这项工作后来在波茨坦天文台 (1942 年)和克里米亚天文台 (1955 年) 也相 继开展起来。除黑子磁场测量外, 海耳也是 太阳普遍磁场研究的先驱。虽然他在这方面 的工作有值得非议之处，但也启发了许多科 学家的继续探索, 成为太阳磁场研究的一个 重要领域。1953 年巴布柯克 (Babcock H. W.) 在海耳太阳实验室创造了太阳磁象仪, 可以测量日面的微弱磁场。这给太阳磁场的

*进一步说，地球的核心以及高空电离层也都是等离 子体。 
研究开辟了一个新纪元。近年来，几架新的 磁象仪的制成导致了日面局部磁场的发现， 为太阳活动现象 (尤其是耀斑)的研究提供了 重要的线索。在最近几年，观测技术及有了 突出的进展。过去只能测量橫向（郎视线方 向)磁场，现在同时能测纵向(垂值于视线方 向）的磁场。这对太阳磁场的研究, 具有极 大的澺义。

既然太阳上存在磁场，多如恆河沙数的 繁星也很可能有磁场。早在 1910 年和 1913 年, 立克天文台的两位科学家就试图进行测 量，但由于技术困难而失败了。后来直到 1946 年，恆星磁场的研究才正式开始。 $\mathrm{H}$. W. 巴布柯克用威耳逊山天文台 100 时反光 镜进行了巨大的工作。他发现了将近 100 颗 “磁星”。它们大都是自旋很快的早型星。在 已知的磁星中, 磁性最強的是 $\mathrm{A}$ 型星 $\mathrm{HD}$ 215441 。它的有效磁场強度竟达 34,400 高 斯。对比起来，太阳的磁性（普遍磁场強度 仅约 1 高斯）真是微不足道了。有趣的是, 所有的磁星都是“磁变星”，師它们的磁场不 断有大幅度的改变。此外, 它们的亮度、视 线速度、谱线轮廓……都有明显的变化。至 于強烈的磁场对磁星的结构和演化肯定有巨 大的影响。这些方面都已有人着手研究。

在大量观测资料的基础上，出现了许多 理论工作。例如黑子的磁场结构、耀理产生
的机制、磁场內辐射转移的理论、磁湍流和 星体等离子体的运动等, 都取得了卓越的成 就。为了总结和交流研究成果, 先后举行了 两次国际性学术会议。这就是 1955 年在斯 德哥尔摩石开的“宇宙物理学中的电磁现象” 讨论会 ${ }^{[1]}$, 以及 1963 年在慕尼黑附近举行 的“恆星和太阳的磁场”会议 ${ }^{[2]}$ 。毫无疑问， 磁场的研究已成为天体物理的一个重要的生 长点。它在人类了解大自然, 征服大自然的 进军途中将发挥忿来愈大的作用。

\section{二 天体的电磁性质}

在讨论天体的磁场以前，首先需要了解 它们的电磁性质。只有这样, 我们才能有的 放矢, 才能断定磁流理论所研究的若干现象 “磁耦合”、“磁场冻结”、“收缩效应”、 “阿耳洨（Alfvén）波”等一一在天体上面是 否存在。代表天体电磁性质最重要的参数是 导电率, 它的表达式为:

$$
\sigma=\frac{\gamma_{e} \cdot 4(2 k T)^{3 / 2}}{\pi^{3 / 2} m^{1 / 2} Z e^{2} L}
$$

此处 $\gamma_{e}$ 为随 $Z$ 变化的系数, $L=2 \ln \left(D / P_{0}\right)$, $D$ 为德拜 (Debye) 牛径, 其他为通用的符 号。由上式可见, $\sigma$ 主要是温度 $(T)$ 的函 数。我们采用文献 [3] 中的数据列成表 1 。 由表可见太阳 (作为恆星的代表)、星际气体 以及电离层的导电率都非常大（一般大于

表 1 天体和电离层的导电率

\begin{tabular}{|c|c|c|c|c|c|c|}
\hline & $\begin{array}{l}\text { 电子滵度 } \\
\left(\text { 厘米 }{ }^{-8}\right)\end{array}$ & $\begin{array}{l}\text { 原子㴽度 } \\
\left(\text { 厘米 }{ }^{-8}\right)\end{array}$ & $\left.{ }^{\text {温 }}{ }^{\circ} \mathrm{K}\right)^{\text {度 }}$ & 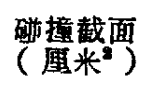 & $\begin{array}{c}\text { 平均自由程 } \\
(\text { (厘米) }\end{array}$ & 导电率 \\
\hline 太阳: 光球 & $3 \times 10^{18}$ & $10^{17}$ & 5800 & $10^{-12}$ & $3 \times 10^{-2}$ & $10^{18}$ \\
\hline 色球 & $10^{10}$ & $10^{10}$ & 6000 & $10^{-12}$ & 60 & $6 \times 10^{12}$ \\
\hline 日冕 & $10^{8}$ & $10^{8}$ & $10^{8}$ & $10^{-16}$ & $10^{8}$ & $7 \times 10^{15}$ \\
\hline 星际气体云：电离氞 & 10 & 1 & $10^{4}$ & $10^{-12}$ & $10^{11}$ & $6 \times 10^{12}$ \\
\hline 中性新 & $10^{-3}$ & 10 & $10^{2}$ & $10^{-8}$ & $10^{11}$ & $7 \times 10^{9}$ \\
\hline 电离层： $F_{2}$ 层 & $10^{8}$ & $2 \times 10^{8}$ & $10^{8}$ & $10^{-10}$ & $10^{4}$ & $3 \times 10^{11}$ \\
\hline $\mathrm{E}$ 层 & $15^{\circ}$ & $10^{18}$ & $3 \times 10^{2}$ & $10^{-9}$ & $10^{4}$ & $2 \times 10^{10}$ \\
\hline
\end{tabular}


$\left.10^{10}\right)$ 。

总的说来，我们面临的问题是电磁现象 与流体运动的结合。这两个范畴的现象，有 时必须合并处理，有时可以分別对待。决定 的制据为下列不等式是否成立:

$$
L H \sigma / \sqrt{\rho}>1
$$

由此可知, 我们研究的对象愈大（師线性长 度 $L$ 大)，磁场 $(H)$ 愈強，导电率 $(\sigma)$ 㨢高， 而密度 $(\rho)$ 愈低, 则上式愈能满足。此时磁 场与流体相互耦合。对行星大气低层 $(\sigma \simeq$ $\left.10^{-3}\right)$ 以及地球上的海洋与湖泊 $\left(\sigma \simeq 10^{10}\right.$, 但 $L$ 很小)，(2)式都不成立。可是对电离层和 天体来说，这个判据却是满足的。例如对太 阳大气, $L H \sigma / \sqrt{\rho} \simeq 3 \times 10^{6} \gg 1$ 。这时显 然存在磁耦合。因此磁场冻结、收缩效应等 一般都成立。这是研究天体磁场的一个重要 的前提。

\section{三 观 测 方 法}

迄今为止，一切天体磁场测量都是利用 天体谱线的塞曼效应进行的。具体说来，都 凭借磁场內辐射的两点性质, 郎 (1) 谱线的 塞曼分裂或变宽，（2）塞曼支线的偏振。一 般使用呈现正常塞曼效应的单重谱线（例如 $\mathrm{Fe} \lambda$ 5250.218)。谱线在磁场內的分裂量 $\left(\Delta \lambda_{H}\right)$ 与磁场強度 $(H)$ 成正比，关系式为

$$
\Delta \lambda_{H}=4.67 \times 10^{-5} g \lambda^{2} \mathrm{H}
$$

此处 $g$ 为谱线的兰德 (Landé) 因子。按上式， 只要测出 $\Delta \lambda_{H}$, 便可计算 $H$ 的数值。测量的 原理就是这样。不过，实际的观测方法却随 研究对象磁场強度的数量级而变。茲分述如 下。

（1）太阳黑子磁场测量装置一一黑子具 有强烈的磁场。已有最高记录达 4,500 高斯, 一般为 2,000-3,000 高斯。这时 Fe $\lambda 5250$ 的
$\Delta \lambda_{H}$ 约为 $10^{-1}$ 埃。对大型摄谱仪来说，这个 数值可以准确测定。具体作法是在摄谱仪狭 㖓前安放 $1 / 4$ 波晶片（使圆偏振光变为平面 偏振光)和偏光膜网(使不同偏振方向的光依 次通过)，于是在底片上得到犬牙交叉的谱 线。这使我们容易直接测出 $\Delta \lambda_{H}$ 。

这个方法简便有效，自从海耳等在威耳 逊山天文台创立后，波茨坦天文台和克里米 亚天文台相继采用，已成为一项长期正规的 观测。测量误差约为 50-100 高斯。 近年 来普耳科沃天交台 ${ }^{[4]}$ 使用中型摄谱仪（分辨 本领 10 万左右)，加上法布里一伯若 (FabryPerot）干涉仪进行黑子磁场测量，已经取得 成效。

（2）磁重仪一一当磁场微弱时，谱线分 裂量太小 (例如对 Fe $\lambda$ 5250，1 高斯磁场所 引起的 $\Delta \lambda_{H}$ 仅约 $4 \times 10^{-5}$ 埃），无法值接测 量。为了观测日面微弱磁场，H.W. 巴布柯 克 ${ }^{[5]}$ 发明了太阳磁象仪。用磁象仪对日面扫 描，便得到磁场的分布图形。

继 H.W. 巴布柯克之后，剑桥大学 ${ }^{[6]}$ 、 克里米亚天文台 ${ }^{[7,8]}$ 、普耳科沃天文台 ${ }^{[9]}$ 和 西德方和裴 (Fraunhofer) 研究所 ${ }^{[10]}$ 相继制成 了磁象仪。虽然这些仪器一脉相承，基本原 理相同，但也各有特色，主要是：（1）克里 米亚磁象仪装有自动的视线速度补偿器, 能 把磁场以外一切原因（太阳自转、日面局部 运动、仪器的机械震动……) 所产生的谱线 位移全部消除。这使磁象仪的记录纯粹代表 磁场強度。（2）普耳科沃的磁象仪几经改进。 最近提出的方案是只用一个狭缝和一个光电 倍增管。这样可以避免许多误差。（3）最近 的一项重大进展是橫向磁场 $\left(H_{\perp}\right)$ 的测量。 过去只能观测纵向磁场 $\left(H_{||}\right)$，很难对整个 磁场強度取得完整的认识。近年来克里米亚 
天交台 ${ }^{[1-13]}$ 对磁像仪作了改装。本来测量 $H_{11}$ 时在狭㖓前面有一个 ADP 晶体, 产生 $O \rightleftarrows \lambda / 4$ 的调频; 现在为了测定 $H_{\perp}$ 加上 一个 $1 / 4$ 波晶片, 作出 $O \rightleftarrows \lambda / 2$ 的调频。 其次, 在测量 $H_{1 \mid}$ 时, 穿过 $S_{1} 、 S_{2}$ 两个狭缝 的光所产生的电流相減; 现在使两个电流相 加。法国天文学家 ${ }^{[14]}$ 用类似的方法测量 $H_{\perp}$, 但不是观测一条谱线, 而是一段连续光谱。 苏联科学院地磁研究所 ${ }^{[15]}$ 根据斯托克斯 (Stokes) 参数 ${ }^{*}$ ，也进行了 $H_{\perp}$ 的测量。

（3）光电磁场记录仪一一这是磁象仪的 雏形。原理与磁象仪相似, 区別为: (1) 光 谱成象处只有一个狭缝; (2) 用旋转偏光膜 代替 ADP 晶体。这种仪器已有三架 ${ }^{[16-18]}$ 。

（4）分光磁场记录仪一一赖赫顿 ${ }^{[19]}$ 独出 心裁地创制了这种仪器, 与太阳单色分光仪 合用，可以粗略记录日面的磁场分布。

以上介绍的都是观测太阳磁场的仪器。 对恆星磁场来说, 问题更为复杂。这是因为 星光很弱, 并且来自恆星圆面各处。假定恆 星磁场为双极的，则各纬度区磁力线与视线 方向的交角 $(\gamma$ ) 不一样 (图 1)。对于一个均

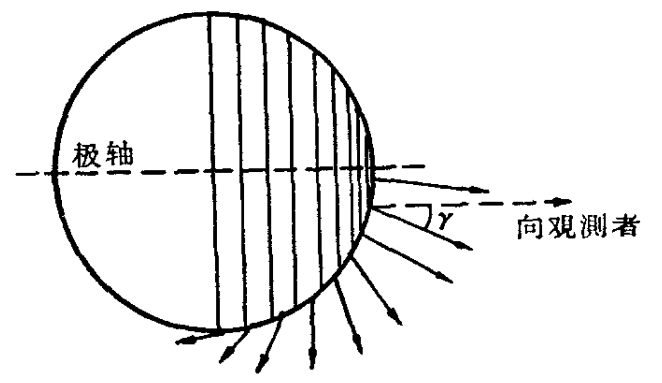

图 1 唒星磁场观测

匀磁化的球体，表面磁场強度 $(H)$ 与纬度 $(\varphi)$ 的关系为:

$$
H=\frac{1}{2} H p\left(1+3 \sin ^{2} \varphi\right)^{1 / 2}
$$

此处 $H_{p}$ 为极区的磁场強度。考虑到临边昏 暗效应, 按 (4) 式对圆面积分, 可知整个恆
星的“有效”磁场強度为 $\mathrm{He}=0.3 \mathrm{Hp}$ 。至于 谱线的三条塞曼支线的強度也与 $\gamma$ 角有关。 关系式为:

$$
\left\{\begin{array}{l}
I_{\sigma_{1}}=\frac{1}{4}(1 \pm \cos \gamma)^{2} \\
I_{\mathrm{II}}=\frac{1}{2} \sin ^{2} \gamma \\
I_{\sigma_{2}}=\frac{1}{4}(1 \mp \cos \gamma)^{2}
\end{array}\right.
$$

在摄谱仪狭缝前面安装 $1 / 4$ 波晶片和方解石 片, 可得到两条偏振方向垂直的谱线。求得 $\Delta \lambda_{H}$ 后，可算出磁场强度。

恆星磁场测量的另一个困难是恆星自转 引起谱线变宽，这使 $\Delta \lambda_{H}$ 很难测量。不幸 的是, 磁场较强的早型星一般都在快速自 转。为了避免这个困难, 只好选择极轴与视 线方向重合的恆星（图 1)。它们的谱线特別 锐细。现有的磁场测量主要是对这些恆星进 行的。

\section{四 太阳黑子磁场}

前面已经谈到, 天体磁场的研究是从太 阳黑子开始的。经过将近 60 年的探索, 我 们对黑子磁场已经取得概略的认识。总的说 来, 由于強磁场的笼笘, 黑子的性质、结构 和运动都与周围的太阳大气迥然不同。因此 有人把黑子叫做日面的“磁性革屿”。海耳等 人的经典性观测, 发现黑子磁场有许多奇异 的特征, 例如 (1) 较大的黑子都是双极的, 先行黑子与后行黑子的磁性相反。(2) 在日 面同一半球，先行黑子和后行黑子分別具有 相同的磁性, 例如前者都是 $\mathrm{N}$ 极而后者都是 $S$ 极的。(3) 日面南北半球的磁性分布相反。 （4）黑子有 22 年的磁周, 即每隔 11 年南北

* 定义见本交第 5 节。 
半球黑子的磁性分布变換一次。应当承认， 这些现象都还沒有得到可靠的解释。

通过谱线的塞曼分裂，只能测出黑子磁 场的強度。为了确定磁场的方向, 需要采用 专门的方法。尼科耳孙利用公式 (5), 由 $\sigma_{1}$ 和 $\sigma_{2}$ 两条支线的強度比决定磁场方向与视线 的夹角 $\gamma_{0}$ 特雷勒尔 ${ }^{[20]}$ 用巴丙湜特 (Babinet) 补偿器测定黑子磁场方向, 精确度较高, 但 技术上困难较大。

有了磁场強度和方向，可以研究黑子的 磁场结构。早已发现，同一黑子各部分的磁 场不一样。以单极黑子而论, 已经推出以下 几个经验公式:

（1）布罗克洪 ${ }^{[21]}$ 公式:

$$
H(r)=H_{0}\left(1-\frac{r^{2}}{b^{2}}\right)
$$

(2) 马提格 ${ }^{[22]}$ 后式:

$$
H(r)=H_{0}\left(1-\frac{r^{4}}{b^{4}}\right) e^{-2(r / b)^{2}}
$$

以上 $H_{0}$ 为黑子中心的磁场强度, $b$ 为黑子 半影的本径, $r$ 为黑子內某一点与中心的距 离。

（3）阿耳洨 ${ }^{[23]}$ 公式:

$$
H(\rho)=\frac{a}{\rho^{3}} \sqrt{1+3 \sin ^{2} \varphi}
$$

此处 $a$ 为偶极磁距, $\rho$ 为向量半径, 后者与 光球表面的交角为 $\varphi_{\text {。 }}$ 无论哪个公式都表 明，黑子磁场強度由中心向边缘減少。至于 磁力线, 它们在黑子上面呈扇形分布。布蒙 $巴^{[24]}$ 用克里米亚磁象仪发现, 磁力线不仅由 黑子中心到边缘向外散开, 而且它们不是直 线，方向逐渐变化。此外，他还认为黑子磁 力线在空间的分布不是均匀的, 而往往汇聚 成磁力线“簇”。

经过改装后，克里米亚磁象仪可以同时 测定纵向和橫向磁场。最近的观测 ${ }^{[13,25]}$ 发现
了黑子磁场一些难于理解的性质。首先, 由 $\lambda 5250$ 线心向外，郎随着光球深度的增加， 偏振面在旋转。旋转的方向既可能是左旋 的，也可能是右旋的。旋转角可接近 $90^{\circ}$ 。 其次，在黑子附近一些小区域內，各种方向 的磁场可以“汼存”。图 2 就是一个实例。图

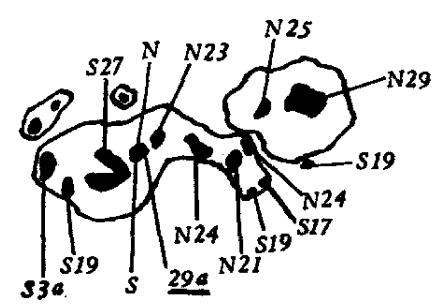

图21961年9月3日黑子的磁场

中一群黑子里有着许多磁场, 既有 $\mathrm{N}$ 极, 也 有 S 极, “a” 表示绝对的橫向磁场, 它们交 织在一起。这些都说明黑子磁场的复杂性。 为了阐明许多引人入胜的特征, 今后还须进 行长期深入的钻研。

\section{五 磁场内的辐射转移理论}

几干高斯的強磁场可能对黑子光谱有某 些影响。这是太阳物理学家长期考虑的问 题。早在 30 年代，已经有人揣测磁场会使 黑子谱线变宽，并使生长曲线变形。但正式 的理论研究直到 50 年代才开始。斯捷潘诺 夫 $^{[26]}$ 和云野 ${ }^{[27]}$ 分別用不同方法, 研究在磁场 內的辐射转移和谱线形成问题, 建立了完整 的逆塞曼效应(郎吸收线的塞曼效应)理论。 我们在下面作简略的介绍。

斯捷潘诺夫用磁光学理论, 求解电子在 磁场中的运动方程

$$
\ddot{\mathbf{r}}+\gamma_{0} \dot{\mathbf{r}}+\frac{e}{m_{c} c} \mathbf{H} \times \mathbf{r}+\omega_{0}^{2} \mathbf{r}=\frac{e}{m} \mathbf{E}
$$

求得磁场內的吸收系数公式为: 


$$
\begin{aligned}
s_{ \pm}= & \frac{1}{2}\left[\left(s_{1}+s_{2}\right)+\frac{1}{2}\left(2 s_{0}-s_{1}-s_{2}\right) \sin ^{2} \gamma \pm\right. \\
& \left. \pm \sqrt{\frac{1}{4}\left(2 s_{0}-s_{1}-s_{2}\right)^{2} \sin ^{4} \gamma+\left(s_{1}-s_{2}\right)^{2} \cos ^{2} \gamma}\right]
\end{aligned}
$$

此处 $s_{0} 、 s_{1} 、 s_{2}$ 分別为正常塞曼效应三条支线的吸收系数, 郎

$$
\begin{aligned}
s_{0} & =\frac{e^{2} \delta_{i k} f_{i k}}{\sqrt{\pi} m_{e} c \Delta \nu_{D}} \int_{-\infty}^{\infty} \frac{\exp \left[-\left(\frac{\nu^{\prime}-\nu}{\Delta \nu_{D}}\right)^{2}\right]}{\left(\nu^{\prime}-\nu_{i k}\right)^{2}+\delta_{i k}^{2}} d \nu^{\prime} \\
s_{1,2} & =\frac{e^{2} \delta_{i k} f_{i k}}{\sqrt{\pi} m_{e} c \Delta \nu_{D}} \int_{-\infty}^{\infty} \frac{\exp \left[-\left(\frac{\nu^{\prime}-\nu}{\Delta \nu_{D}}\right)^{2}\right]}{\left[\nu^{\prime}-\left(\nu_{i k} \pm \Delta \nu_{0}\right)\right]^{2}+\delta_{i k}^{2}} d \nu^{\prime}
\end{aligned}
$$

$s_{+}$和 $s_{-}$是两种互为共轭偏振的辐射的吸收 系数。可以认为在太阳大气中有偏振方向互 为共轭的两个辐射场 $I_{+}$和 $I_{-}$, 以及吸收系 数各为 $s_{+}$和 $s_{-}$的两类原子。这两类原子分 別只吸收 $I_{+}$和 $I_{-}$辐射。这使磁场內辐射转 移的问题大为简化, 因为我们可以对两种辐 射和两类原子写出两个转移方程:

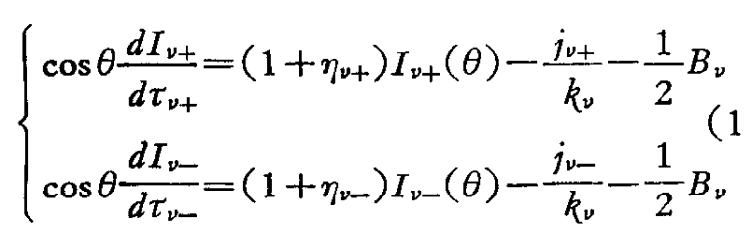

这与一般转移方程的差异, 只是能源函数不 是 $B_{\nu}$ 而为 $\frac{1}{2} B_{\nu}$, 因此已有的各种解法都可 朵用。把求出的结果按公式 $I_{\nu}=\frac{1}{2}\left(I_{\nu+}+\right.$ $I_{\nu}$ ) 合并起来，斯捷潘诺夫发现:（1）对不 同的 $\gamma$ 角, 谱线轮廓大不一样。(2) 磁场使 谱线的等值宽度 $\left(W_{1}\right)$ 增加。 $H$ 和 $\gamma$ 愈大时, 增加量 $\Delta W$ ，都愈大。（3）磁场对生长曲线的 影响是使过渡段上升，但对线性段和阻尼段 影响很小。

云野利用斯托克斯参数研究逆塞曼效 应。四个参数的定义为 $I \equiv I_{1}+I_{2}, Q \equiv I_{1}-$ $I_{2}, U \equiv 2 \xi_{1} \xi_{2} \cos \left(\epsilon_{1}-\epsilon_{2}\right), V \equiv 2 \xi_{1} \xi_{2} \sin \left(\epsilon_{1}-\right.$ $\left.\epsilon_{2}\right)$, 此处 $\xi_{1}$ 和 $\xi_{2}$ 分別为光线的电向量在 $x$
和 $y$ 轴上的分量, 而 $\epsilon_{1}$ 和 $\epsilon_{2}$ 是它们的相位 角。用这四个参数可以确定辐射強度、偏振 度、偏振面的方向以及偏振光的椭率等四个 物理量。斯托克斯参数的特点是: 当几束偏 振光混合时, 合成光的任一参数 (如 $U$ ) 为各 束偏振光的相应参数之和 (郎 $U=U_{1}+U_{2}+$ …)。这使磁场內偏振光的传播问题大为简 化。

比较起来, 斯捷潘诺夫的方法简便实 用, 而云野的理论严谨, 具有较大的普遍 性。有趣的是, 拉奇科夫斯基 ${ }^{[28]}$ 证明了这两 套理论在实质上是一样的, 它们的方程组可 以互相归化。

\section{六 日面局部磁场}

磁象仪的观测，发现太阳表面的局部区 域 (一般在黑子附近的活动区) 有着较強的磁 场, 强度约为 10-10 2 高斯。由磁象仪的描 记图, 可以看出局部磁场的磁性、磁场強度 以及等磁強线的分布情况。总的说来，局部 磁场的结构极为复杂; 它们往往是多极的, 不规则的和相互联系的。早在 19 世纪末期 天文学家已经由 $H_{a}$ \& $\mathrm{KCa}$ II 单色象发现, 色球具有极为复杂的精细结构。把磁象仪记 录与色球单色象对照, 就可一目了然地看出 
二者甚为相似。这告诉我们, 色球物质的分 布和运动主要受磁场支配。进一步说，色球 精细结构由磁场决定的条件是

$$
\frac{H^{2}}{8 \pi} \geqslant \frac{1}{2} \rho v^{2}
$$

对色球取 $\rho \simeq 10^{-10}$ 克/厘米 ${ }^{3}, v \simeq 10^{5}$ 厘米/秒，则得磁场強度的下限仅为 $H_{0} \simeq 1$ 高斯。这说明微弱的局部磁场已经可以使色 球物质“唯命是听”，沿磁力线排列。

局部磁场的发现导致了一个重大课题 ——耀斑产生机制一一的研究。我们知道,

耀玟是最強烈的太阳活动现象。一次大耀斑 爆发可以释放出 $10^{31}-10^{33}$ 尔格的巨大能量, 这比整个色球和日睌所矰藏的全部能量还 多! 这个能量从何而来? 天文学家早已开始 寻求这一问题的答案。不少人认为, 磁能的 转化是一个可能的途径。具体说来，在耀斑 区域几百高斯的磁场一旦“全军覆灭”，就能 放出 $10^{30}$ 尔格的巨额能量。因此，耀斑可能 是由磁场变化所引起的。

十几年前已经有人提出耀斑的中和点理 论, 认为在中和点 (郎局部磁场強度为零的 区域）附近磁场不稳定，机械力量可使等离 子体压缩，由此产生耀斑。谢维尔尼 (Cеверный А. Б.) 发展了这个理论。他用磁象仪 记录，发现耀斑出现区域有较大的磁场梯 度，而在耀玫出现后，梯度減小，磁场结构 显著简化。他认为较大的磁场梯度产生的机 械力，使等离子体向中和点压缩。如果压缩 速度超过声速，就出现冲击波。冲击波的碰 撞 (郎所谓的 “收缩效应”) 产生 $10^{6}-10^{7}{ }^{\circ} \mathrm{K}$ 的高温, 发生某些核反应, 形成高能质点、 宇宙射线和 X辐射。換言之, 耀斑爆发意味 着䅔藏在太阳磁场中的能量迅速获得解放。 不少天文工作者 ${ }^{[29-31]}$ 的观测证实了谢维尔尼
的论断，郎耀斑出现后局部磁场显著变形。 但是也有人 ${ }^{[32,33]}$ 得出否定的结果，郎在耀斑 发生前后磁场并无明显变化。因此, 我们不 能认为耀斑产生的机制问题已经圆满解决。

\section{七 太阳普逼磁场}

长期的太阳观测，发现的不少现象（例 如日菟辐射带由两极伸出，指向低纬区; 太 阳黑子有 22 年的磁周…) 使人想到，太阳 和地球相似，具有普遍磁场。海耳等 ${ }^{[6]}$ 从 1912 年起致力于太阳普遍磁场的研究。他们 认为太阳有一个和地球相似的均匀的偶极磁 场。于是表面磁场强度与纬度的关系由 (4) 式决定。磁力线的倾角为 $\delta=\tan ^{-1}(2 \tan \varphi)$ 。 就视线方向而言, 磁场強度 $\left(H_{\nu}\right)$ 与纬度的 关系如图 3 所示。海耳等对某些谱线确实观

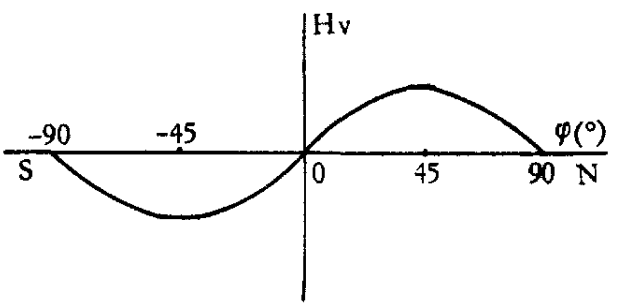

图 3 日面视向磁场随纬度的变化

测到这种关系。他们求得太阳极区的磁场強 度约为 25-50 高斯; 关且和地磁场相似, 南(北)磁极位于旋转轴的北(南)极附近, 磁 轴与旋转轴有 $6^{\circ}$ 的交角。

继海耳之后，许多天文工作者从事太阳 普遍磁场的观测。他们发现海耳的结果是值 得怀疑的。太阳普遍磁场不一定存在，郎使 存在也只有 $1-2$ 高斯的強度, 这远小于海 耳的数值。这方面工作的概况可从表 2 看 出。

有趣的事情是太阳普遍磁场的磁极在 1957 -58 年变号。这是 H. D. 巴布柯克 ${ }^{[35]}$ 
表 2 太阳普遍磁场观测

\begin{tabular}{|c|c|c|c|c|c|c|c|c|}
\hline 份 & 观 & 测 & 者 & 仪 & 分辦本领 & \multicolumn{2}{|c|}{ 记录方法 } & 极区磁场強度(高斯) \\
\hline 1913 & \multicolumn{3}{|l|}{ 海耳 } & 光 棚 & 20 万 & 照 & 相 & $\leqslant 50$ \\
\hline $1933-34$ & \multicolumn{3}{|c|}{ 尼科耳孙等 } & 光 棚 & 20 万 & 目 & 视 & $3.6 \pm 1.7$ \\
\hline 1944 & \multicolumn{3}{|c|}{ 冯·克鲁伯(von Klüber H.)等 } & 法布里一伯若干涉仪 & 35 万 & 照 & 相 & $\ll 10$ \\
\hline 1948 & \multicolumn{3}{|c|}{ 席生 (Thiessen G.) } & 法布里-伯若干涉仪 & & 目 & 视 & $1.4 \pm 3.3$ \\
\hline 1950 & \multicolumn{3}{|c|}{ 冯·克鲁伯 } & 吕麦 (Lummer) 片 & 60 万 & & 相 & $<1-2$ \\
\hline 1952 & \multicolumn{3}{|c|}{ H.W.巴布柯克等 ${ }^{[5]}$} & 磁象仪 & 60 万 & & 记录 & 很弱 \\
\hline 1955 & \multirow{2}{*}{\multicolumn{3}{|c|}{$\begin{array}{l}\text { H. D. 和 H. W. 巴布柯克等 }{ }^{[34]} \\
\text { 谢维尔尼[25] }\end{array}$}} & 磁象仪 & 60 万 & \multicolumn{2}{|c|}{ 光电记录 } & $\sim 1$ \\
\hline 1963 & & & & 磁象仪 & 45 万 & \multicolumn{2}{|c|}{ 光电记录 } & 1.6 (北极)；0.1(南极) \\
\hline
\end{tabular}

用磁象仪作出的重要发现。根据他的观测, 太阳几何南极区的磁场在 1957 年 3 月至 7 月逐渐由 $\mathrm{S}$ 极变为 $\mathrm{N}$ 极，而几何北极区的磁 场在 1958 年 11 月內甚至突然地由 $\mathrm{N}$ 极变为 $\mathrm{S}$ 极。在这以前，按 H. D. 和 H. W. 巴布 柯克父子 ${ }^{\left[{ }^{[3]}\right.}$ 的观测，太阳普遍磁场与地球磁 场的磁性相反，但现在二者趋于一致了。值 得注意，太阳普遍磁场的磁性反号不是在南 北两极同时发生的。在 1957 年年中到 1958 年末期的一年半內，两极都是 $\mathrm{N}$ 极。这是一 个奇怪的现象，给太阳磁场的研究留下一个 有趣而困难的题材。

\section{八恒星的磁场}

本文第一节已经谈到，恆星磁场的研究 是从 1946 年正式开始的。H.W. 巴布柯克 ${ }^{[36]}$ 用与黑子磁场观测类似的方法，进行了巨大 的工作，给出了 89 颗磁星的表 ${ }^{[37]}$ 。这些星 的有效磁场強度一般为几百高斯，但也有強 达 5,000 多高斯的。它们的星型区分见表 3。

表 3 磁星的型別

\begin{tabular}{l|cc}
\hline \multicolumn{1}{c|}{ 型 } & 別 & 星 \\
\hline A 型锐线星 & \multicolumn{2}{|c}{70} \\
金属线星 & \multicolumn{2}{|c}{7} \\
M型巨星 & \multicolumn{2}{|c}{3} \\
S 型星 ( 双子座 R 星和 HD 1105) & 2 \\
星团变星 ( 天琴座 RR 星 ) & 1 \\
\hline
\end{tabular}

值得注意的是，绝大部分磁星是 $\mathrm{A}$ 型星。这 些恆星又可按磁场強度的变化情况而分为 $\alpha 、 \beta 、 \gamma$ 三类(分別以猎犬座 $\alpha^{2}$ 、北莬座 $\beta$ 、 小马座 $\gamma$ 三颗星作为典型）。 $\alpha$ 型磁星作周 期性的磁场強度变化，但強度和相位有时与 平均值相差甚大。 $\beta$ 型磁星变化不规则, 不 仅強度在变，磁性也会反转。至于 $\gamma$ 型磁星 也呈现不规则的磁场強度变化，但磁性不会 转換。我们谈到过, 已知最強的磁星是 $H D$ 215441 。这就是一颗 $\gamma$ 型磁星。它的磁场強 度最大可达 34,400 高斯，而最小值为 4,140 高斯。对一般磁星来说, 磁场強度的变幅自 然小得多、大约为 $1,000-2,000$ 高斯。

除磁场強度外, 磁星的谱线強度和轮 廓、元素丰度、视线速度、亮度以及光线的 偏振度都在变化。在这些方面有许多复杂的 现象（详细描述见 Gollnow H. 在交献 [2] 中的第二篇报告)，主要是：（1）各元素谱线 強度的变化情况不同。例如当猎犬座 $\alpha^{2}$ 星的 负极磁场达到极大时, EuII 谱线最亮; 而当 正极磁场最強时， $\mathrm{Cr}$ 线的亮度增到极大值。 可是对 HD 125248 星，情况正好相反。（2） 各个磁星的化学成分不同。例如猎犬 $\alpha^{2}$ 的稀 土元素特別丰富, 但有的磁星却连一条稀土 元素谱线也沒有。（3）似乎一切磁星的亮度 都在变化，不过变幅很小，一般约百分之几 星等。值得注意, 对 $\alpha$ 型磁星来说, 磁性周 
期与光度周期相同, 只是对有的磁星来说, 光度极大与正极磁场最强时间相符; 而对另 一些磁星，都与负极磁场的极大同时发生。 所有这些现象都还沒有得到可靠的解释。要 深刻了解恆星磁场的本质，天文学家还须付 出大量的、辛勤的劳动。

附带谈到，除太阳和恆星外，广阔无垠 的星际空间也具有磁场。主要的证据 是:

(1)银河系发出射电辐射，这不全是热辐射。 产生非热射电辐射的机制无论是等离子体振 荡, 或是相对论性电子在磁场中的运动, 都 与磁场有关。(2) 星光有偏振现象, 偏振面 接近黄道。（3）弥漫星云大都呈长条形, 和 磁力线相似。这很难认为是银河系自转造成 的，因为银河自转需要 5 千万年才能使太阳 附近的物质集团变长一倍, 这不足以说明弥 漫云的长度。一种很自然的解释是星际磁场 的影响。归纳起来，用各种方法求得的星际 磁场強度约为 $10^{-5}-10^{-6}$ 高斯。这自然是极 度微弱的磁场。但考虑到星际空间的辽阔广 大，我们可以想到星际磁场的存在对天体的 起源和演化会有重大的影响。

\section{九 天体磁场的起源}

太阳和恆星的磁场是怎样产生的? 这还 是一个远未得到解决的难题。不少天文工作 者从事这方面的探索，提出了形形色色的学 说。归纳起来, 已有的理论不外乎两个范 畴。一些天文学家 ${ }^{[3,393}$ 认为, 恆星磁场是形 成恆星的原始物质的磁场遗留下来的。換言 之，恆星磁场是先天具备的。但是以考 灵 (Cowling T. G.) $)^{[2,401}$ 为主的另一些学者却 主张恆星磁场是后天形成的, 系由恆星的自 转、对流等运动所造成的“电动机效应”的产 物。目前还很难判断这两种学说就是孰非。
不过, 如果考虑到 $\mathrm{A}$ 型星自转较快, 对流层 较厚, 而它们的磁场最強, 我们可以相信第 二类假说至少在一部分情况下是符合实际 的。

进一步说，第一类学说只是把恆星磁场 起源的问题推到另一方面，而并沒有解决问 题。正如考灵 ${ }^{[2]}$ 最近指出的, 星际气体云的 磁场也由带电质点的运动产生; 因此可以认 为, 一切的天体磁场起源学说归根到底都以 “电动机效应”为基础。这意味着，天体的磁 场是与它们的运动密切联系着的, 在这里需 要提到, 辩证唯物主义告诉我们, 世界上存 在着的只是运动着的物质。睡然宇宙中绝大 部分物质都是等离子体，而运动着的等离子 体都具有磁场，因此磁场的研究可以启发我 们了解天体运动状态的实质。这可以说是天 体磁场研究的根本意义。

[1] Lehnert B. (ed.), "Electromagnetic Phenomena in Cosmic Physics", Cambridge Univ. Press (1958).

[2] Lüst R., "Stellar and Solar Magnetic Fields", North-Holland Publ. Co. (1965).

[3] Пикельнер С. Б., “Основы космической электродинамики", Ф-М (1961).

[4] Вяльшин Г. Ф., Солнечные данные, № 5, 143 (1956); № 1, 143 (1957); № 2, 116 (1957).

[5] Babcock H. W., Ap. J., 118, 387 (1953).

[6] Beggs D. W., von Klüber H., Nature, 178, 1412 (1956).

[ 7 ] Никулин Н. С., Северный А. Б., Степанов В. Е., Изв. КрАО, 19, 3 (1958).

[8] Никулин Н. С., Изв. КрАО, 22, 3 (1960).

[9] Котляр Л. М., Изв. ГАО, № 163, 73 (1963); Астр. жур., 37, 469 (1960).

[10] Deubner F. L., Kiepenheuer K. O., Liedler D., Z. Astrophysik, 52, 118 (1961).

[11] Степанов В. Е., Северный А. Б., Изв. КрАО, 28, 166 (1962).

[12] Никулин Н. С., Изв. КрАО, 31, 209 (1964).

[13] Северный А. Б., Изв. КрАО, 31, 126 (1964).

[14] Leroi J. L., "Contribution à l' étude de la polarisation de la lumière solaire" (1962), Thesis, Paris. 
[15] Иошпа Б. А., Обридко В. Н., Геомагнетизм и аэрономия, 2, 541 (1962); А. Ж., 40, 1013 (1963); Геомагнетизм и аэрономия, 4, 17 (1964).

[16] Thiessen G., Z., Astrophysik, 30, 185 (1952).

[17] Kiepenheuer K. O., Ap. J., 117, 447 (1953).

[18] Могилевский М. А., Веллер А. Е., Вальд-Перлов В. М., ДАН, 95, 957 (1954).

[19] Leighton R. B., Ap. J., 130, 366 (1959).

[20] Treanor P. J., M. N., 120, 412 (1960).

[21] Broxon J. W., Phys. Rev., 62, 521 (1942).

[22] Mattig W., Z. Astrophysik, 31, 273 (1953).

[23] Alfvèn H., "Cosmical Electrodynamics", Oxford (1950).

[24] Бумба В., Изв. КрАО, 23, 212 (1960).

[25] Severny A., Space Science Reviews, 3, 451 (1964).

[26] Степанов В. Е., Изв. КрАО, 18, 136 (1958); 19, 20 (1958).

[27] Unno W., Publ. Astr. Soc. Japan, 8, 108 (1956).

[28] Рачковский Д. Н., Изв. КрАО, 25, 277 (1961); 26, 63 (1961).
[29] Бумба В., Изв. КрАО, 19, 105 (1958).

[30] Bruzek A., Z. Astrophysik, 50, 110 (1960).

[31] Antalova A., Bull. Astr. Inst. Czechosl., 12, 108 (1961).

[32] Howard R., Babcock H. W., Ap. J., 132, 218 (1960).

[33] Michard R., Mouradin Z., Senul M., Ann. Astrophysique, 24, 54 (1961).

[34] Babcock H. W., Babcock H. D., Ap. J., 121, 349 (1955).

[35] Babcock H. D., Ap. J., 130, 364 (1959).

[36] Babcock H. W., Ap. J., 105, 105 (1947); P.A.S.P., 59, 112 (1947).

[37] Babcock H. W., Ap. J. Suppl., 3, No. 30 (1958); Chap. 7 in "Stellar Atmospheres" (ed. J. I. Greenstein) (1960).

[38] Гуревич Л. Э., В сб. “Вопросы космогонии", 2, 150 (1954).

[39] Mestel L., Spitzer L., M. N., 116, 503 (1956).

[40] Cowling T. G., in "Vistas in Astronomy" (ed. A. Beer), 1, 313 (1955). 